\title{
Massive subchorionic haemorrhage: a rare case report associated with secondary PPH due to uterine artery pseudoaneurysm
}

\author{
J. Arumaikannu*, S. Usha Rani, T. S. Aarifa Thasleem
}

Institute of Obstetrics and Gynecology, Egmore, Chennai, Tamil Nadu, India

Received: 08 August 2017

Accepted: 04 September 2017

\section{*Correspondence:}

Dr. J. Arumaikannu,

E-mail: drjak07@yahoo.co.in

Copyright: (C) the author(s), publisher and licensee Medip Academy. This is an open-access article distributed under the terms of the Creative Commons Attribution Non-Commercial License, which permits unrestricted non-commercial use, distribution, and reproduction in any medium, provided the original work is properly cited.

\begin{abstract}
Massive subchorionic hemorrhage is a rare but serious condition in pregnancy in which a large amount of blood, mainly maternal collects between the uterine wall and the chorionic membrane and may leak through the cervical canal. Although many associations have been reported, an underlying etiology has not been elucidated. Association of massive subchorionic hemorrhage with thrombophilias have been reported in few articles. We are reporting a case of massive subchorionic hemorrhage presented at 13 weeks of gestation associated with secondary post-partum hemorrhage due to uterine artery pseudoaneurysm.
\end{abstract}

Keywords: Massive subchorionic haemorrhage, Secondary PPH, Uterine artery pseudoaneurysm

\section{INTRODUCTION}

During pregnancy, minor degrees of haemorrhage on the chorionic plate surface of the placenta are commonly identified on ultrasound assessment. Hemorrhage beneath or at the edge of the placenta, or behind an isolated area of the fetal membrane is of greater concern. Hemorrhage on the chorionic plate surface of the placenta are commonly identified on ultrasound assessment and is usually asymptomatic. Since is on the placental surface it cannot escape to cause vaginal bleeding. By contrast hemorrhage at the edge of the placenta or behind an isolated area of the fetal membrane, blood may track externally to cause vaginal bleeding. ${ }^{1}$

Later in the first trimester and early second trimester, the subchorionic hematoma partially strips the developing placenta away from its attachment site. Therefore prognosis of patients with type of hematoma in second trimester is worse than the prognosis of patients with hematoma early in first trimester. Massive subchorionic hemorrhage in the second trimester may also compromise maternal health. ${ }^{2,3}$

A subchorionic hemorrhage can be considered large or massive if it is greater than $50 \%$ of the size of the gestational sac, medium if it is $20-50 \%$ and small if it is less than $20 \%$. $^{2,3} \mathrm{We}$ report here a case of massive subchorionic hemorrhage at 13 weeks of gestation.

\section{CASE REPORT}

A 29 years old South Indian origin woman gravida 4 para 2 live 2 abortion 1 with no medical comorbidities, with two term previous uneventful caesarean deliveries and one previous incomplete abortion at 11 weeks gestation for which dilatation and curettage was done had admitted with vaginal bleeding at 13 weeks 6 days of gestation in the present pregnancy. On examination vitals were stable and uterus was just palpable per abdomen. USG showed an alive intrauterine gestation with retro placental hematoma of size $3.6 \times 2.8 \mathrm{~cm}$ in posterior wall of the 
uterus. Routine investigation showed hemoglobin 7.6 $\mathrm{g} / \mathrm{dl}$. Patient was transfused two units of packed red blood cells. Three hours after admission patient had tachycardia (HR- 120-130/min) and blood pressure of 80/50 $\mathrm{mmHg}$. On examination uterus had enlarged to 24 weeks size, USG done showed subchorionic hemorrhage of $10 \times 10$ $\mathrm{cm}$ occupying the entire posterior wall of her uterus. Hemoglobin was $5 \mathrm{gm} / \mathrm{dl}$. Owing to sudden increase in the size of subchorionic hemorrhage and hemodynamic instability, hysterotomy was proceeded.

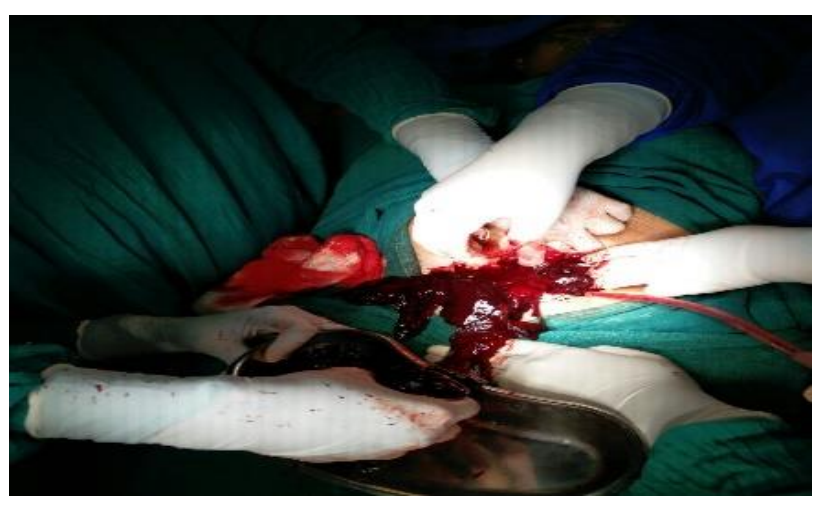

Figure 1: Intraoperative pictures showing massive subchorionic hemorrhagic clots.

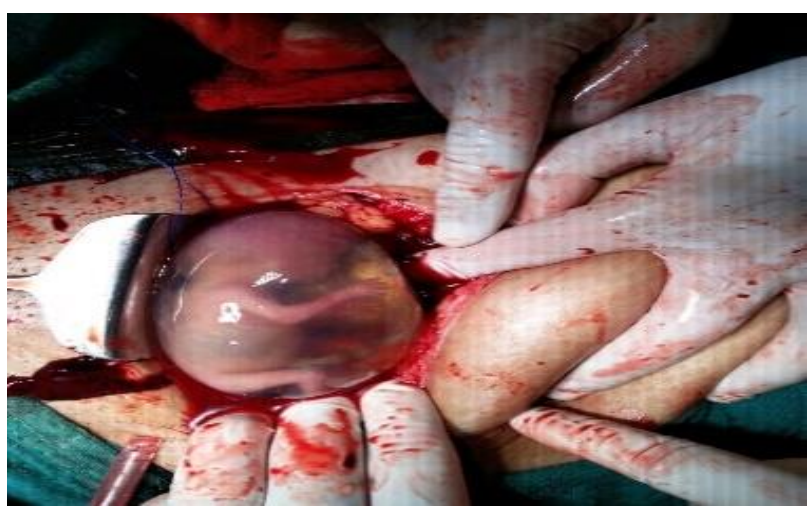

Figure 2: intraoperative picture showing an alive fetus within an intact sac.

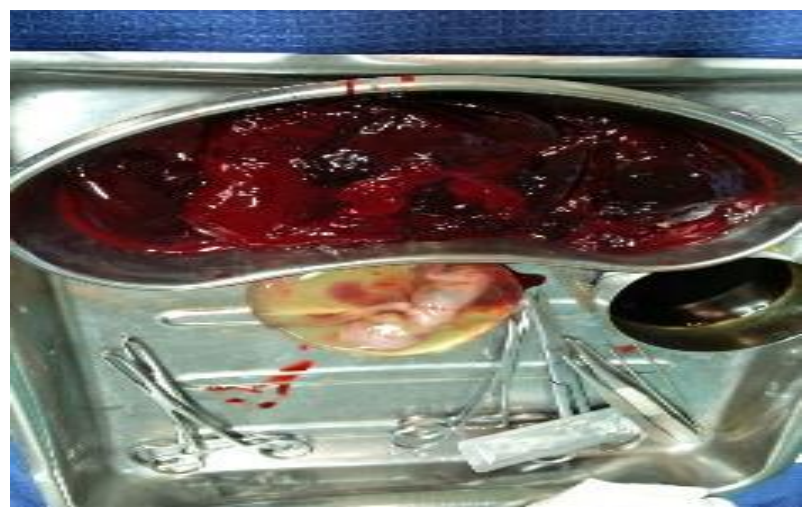

Figure 3: In vitro picture showing $\mathbf{5 5 0}$ grams of subchorionic clots and an alive fetus.
Intra op findings showed the uterus was enlarged to 24 weeks size. Hysterotomy incision was made in the lower segment of the uterus. A massive hematoma occupying almost the entire uterine cavity of size $15 \mathrm{~cm} \times 10 \mathrm{~cm}$ pushing the amniotic sac with an alive fetus to one corner was noted. An alive fetus with intact sac was delivered. The hematoma of about 550 grams was evacuated. Uterine cavity was emptied. The uterus was found to be anatomically normal.

\section{Intraoperative pictures}

Perioperatively 4 units of packed red blood cells and 6 units of fresh frozen plasma was transfused.

After 3 hours of surgery patient had an episode of vaginal bleeding and about 300 grams of clots removed. Intrauterine balloon tamponade was done to control bleeding for 24 hours. Following these 2 units of packed red blood transfusion was done.

Lupus anticoagulant, Antithrombin III, Protein C, Protein $\mathrm{S}$, ANA and ACL were found to be negative.

MRI pelvis done on $10^{\text {th }}$ postoperative day: axial gradient -echo T1 weighted MR angiogram demonstrated flow void in right uterine artery (arrowhead) due to turbulent flow. Impression: right uterine artery pseudoaneurysm.

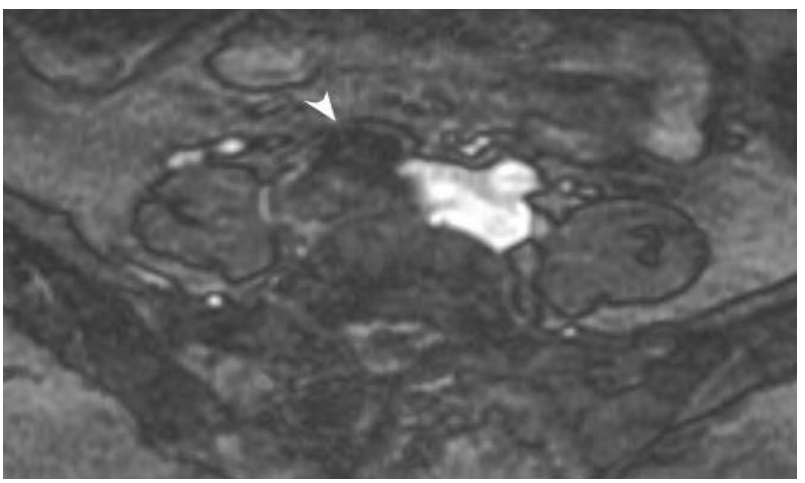

Figure 4: T1 weighted MR angiogram showing flow voids in right uterine artery.

On $11^{\text {th }}$ post-operative day patient had another episode of massive secondary post-partum hemorrhage. On examination: HR- 146/min, BP- $60 \mathrm{mmHg}$, Sp02- 90\% with oxygen. Abdomen was soft and uterus was involuting, per vaginal examination revealed profuse bleeding.

Resuscitative measures initiated. Medical management for post-partum hemorrhage was attempted. Profuse Bleeding through vagina was persistent despite effective medical management. Patient went in for hypovolemic shock. 4 units of packed red blood cells and 6 units of fresh frozen plasma were transfused. Inotropes started. In view of persistent vaginal bleeding, proceeded with emergency laparotomy 
Abdominal exploration revealed a uterus of normal size and normal adnexa without intra-abdominal bleeding. After the peritoneum of the urinary bladder was detached, the cesarean scar was inspected and it was found to be healthy. The source of high-flow bleeding was found to be the aneurysmatic formation associated with the right uterine artery, within the uterine cavity. Hence proceeded with hysterectomy.

Histopathogical examination: endometrium and myometrium shows normal gestational changes. Postoperative period after hysterectomy was uneventful.

\section{DISCUSSION}

Subchorionic hematomas are common and insignificant sonographic findings in patients with vaginal bleeding in weeks 9-20 of pregnancy. The incidence rate has been reported between $1.3-62 \%$ depending on the patient population that was studied. ${ }^{4-7} \mathrm{~A}$ number of studies have investigated the association between the presence of subchorionic hemorrhage and adverse pregnancy outcomes. Nonetheless, the clinical significance of subchorionic hemorrhage and its effects on pregnancy outcomes remains controversial. Ultrasound examinations are indicated to assess the viability of a pregnancy in the context of vaginal bleeding in the second trimester, but it is rare to observe hematomas that are larger than the fetus. Here we report one such case where subchorionic hemorrhage is much larger than the fetus.

Placenta of this case showed normal morphological features and normal echogenic texture in ultrasonogram. Case reports of massive intrauterine hematomas describe poor outcomes when the hematoma is associated with the placental pathology, probably because this represents a variant of abruption. 8,9 They confirm that abnormalities of the placenta, especially placentomegaly, constitute a poor prognostic sign and suggest abruption from underlying chronic uteroplacental vascular disease. Placental evaluation may therefore be of prognostic significance in women whose pregnancies are found to have significant hematomas in the second trimester.

Initial size in relation to gestational sac size at presentation (worse if large) and location (worse prognosis if retro-placental) determines outcome. In our case the hemorrhage was retroplacental and behind the fetal membrane which itself indicates poor prognostic sign.

The main reasons for vaginal bleeding in early gestation are subchorionic hemorrhage and rupture of a marginal placental sinus. Its etiology is largely unknown, although uterine malformations, history of repeated abortions and infection have been suggested as possible predisposing factors. Though no uterine malformations were noted, history of one abortion is present in our patient and it could have been a predisposing factor for subchorionic hemorrhage.

The size of the hematoma is graded according to the percentage of chorionic sac circumference elevated by the hematoma as follows. Small indicates less than one-third of the chorionic sac circumference elevated by hematoma. Moderate indicates one-third to one-half of the chorionic sac circumference elevated by hematoma. Large indicates one-half to two-thirds or greater of the chorionic sac circumference elevated by hematoma. The size of these hematomas understandably could result in discussions about the wisdom of pregnancy continuation and maternal safety.

Since case reports of sub chorionic hematoma have been reported with patient's positive to ANA ACL antibodies, Lupus anticoagulant, Protein S and Protein C, these tests for thrombophilia were done and found to be negative. ${ }^{10}$

Our patient also developed secondary postpartum hemorrhage with MRI findings of right uterine artery pseudoaneurysm. Pseudoaneurysm of the uterine artery is an uncommon cause of delayed postpartum hemorrhage. Trauma to the uterine artery during surgery may cause a defect in the arterial wall, through which arterial blood escapes and diffuses to the adjacent tissues, resulting in the formation of a hematoma. When this hematoma is in continuity with the uterine artery that supplies continuous blood flow, a pseudoaneurysm forms. The absence of a three-layered arterial wall lining in a pseudoaneurysm differentiates it from a true aneurysm. The development of an arterial pseudoaneurysm is a rare but reported complication of pelvic surgery, vascular trauma during hysterotomy, C- section or after uterine curettage. ${ }^{11}$ Uterine curettage done for previous abortion in this patient may be a predisposing factor for the development of pseudoaneurysm.

A uterine artery embolization procedure was scheduled the day after the diagnosis of pseudoaneurysm. However, on the day of the intervention, the patient experienced excessive vaginal bleeding (approximately $1500 \mathrm{~mL}$ ) and underwent emergency laparotomy.

Funding: No funding sources

Conflict of interest: None declared

Ethical approval: Not required

\section{REFERENCES}

1. Windrim C, Athaide G, Gerster T, Kingdom JC. Sonographic findings and clinical outcomes in women with massive subchorionic hematoma detected in the second trimester. J Obstet Gynecol Can. 2011;33(5):475-9.

2. Chhabra A, Lin EC. Subchorionic Hemorrhage Imaging. Available at: http://emedicine.medscape. com/article/404971-overview. Accessed on 3 June 2017. 
3. Leite J, Ross P, Rossi AC, Jeanty P. Prognosis of Very Large First-Trimester Hematomas. J Ultrasound Med. 2006;25:1441-5.

4. Ball RH, Ade CM, Schoenborn JA, Crane JP. The clinical significance of ultransonographically detected subchorionic hemorrhages. Am J Obstet Gynecol. 1996;174(3):996-1002.

5. Nagy S, Bush M, Stone J, Lapinski RH, Gardó S. Clinical significance of subchorionic and retroplacental hematomas detected in the first trimester of pregnancy. Obstet Gynecol. 2003;102(1):94-100.

6. Pedersen JF, Mantoni M. Prevalence and significance of subchorionic hemorrhage in threatened abortion: a sonographic study. AJR Am J Roentgenol. 1990;154(3):535-7.

7. Ylöstalo $\mathrm{P}$, Ammälä $\mathrm{P}$, Seppälä M. Intrauterine haematoma and placental protein 5 in patients with uterine bleeding during pregnancy. $\mathrm{Br} \mathrm{J}$ Obstet Gynaecol. 1984;91(4):353-6.

8. Gupta R, Sharma R, Jain T, Vashisht S. Antenatal MRI diagnosis of massive subchorionic hematoma: a case report. Fetal Diagn Ther. 2007;22:405-8.
9. Kojima K, Suzuki Y, Makino A, Murakami I, Suzumori K. A case of massive subchorionic thrombohematoma diagnosed by ultrasonography and magnetic resonance imaging. Fetal Diagn Ther. 2001;16:57-60.

10. Heller DS, Rush D, Baergen RN. Subchorionic hematoma associated with thrombophilia: report of three cases. Pediatr Dev Pathol. 2003;6(3):261-4.

11. Kwon JH, Kim GS. Obstetric iatrogenic arterial injuries of the uterus: Diagnosis with US and treatment with transcatheter arterial embolization. Radiographics. 2002;221:35-46.

12. Fung TY, To KF, Sahota DS, Chan LW, Leung TY, Lau TK. Massive subchorionic thrombohematoma: a series of 10 cases. Acta Obstet Gynecol Scand. 2010;89:1357-61.

Cite this article as: Arumaikannu J, Rani SU, Thasleem TSA. Massive subchorionic haemorrhage: a rare case report associated with secondary $\mathrm{PPH}$ due to uterine artery pseudoaneurysm. Int J Reprod Contracept Obstet Gynecol 2017;6:4723-6. 